\title{
Arabidopsis mutants define downstream branches in the phototransduction pathway
}

\author{
Hsou-min Li, Lothar Altschmied, ${ }^{1}$ and Joanne Chory ${ }^{2}$ \\ Plant Biology Laboratory, The Salk Institute, San Diego, California 92186-5800 USA
}

\begin{abstract}
Light regulates the development of Arabidopsis seedlings in a variety of ways, including inhibition of hypocotyl growth and promotion of leaf development, chloroplast differentiation, and light-responsive gene expression. Mutations that uncouple most or all of these responses from light control have been described, for example, $\operatorname{det} 1, \operatorname{det} 2$, and cop 1 . To identify regulatory components that define downstream branches in the light-regulated signal transduction pathway, mutants specifically affected in only one light-regulated response were isolated. A screen was designed to isolate mutants that overexpressed the $C A B$ (photosystem II type I chlorophyll $a / b$-binding proteins) genes in the dark, by use of a transgenic line containing a T-DNA construct with two $C A B 3$ promoter-reporter fusions. Eight mutants that showed aberrant expression of both $C A B 3$ promoters were isolated and were designated doc mutants (for dark overexpression of $C A B$ ). All of the mutants have normal etiolated morphology in the dark. Genetic and phenotypic analyses indicate that most of the mutations are recessive and define at least three loci $(\operatorname{doc} 1, \operatorname{doc} 2, \operatorname{doc} 3)$. Unlike $\operatorname{det} 1$ and $\operatorname{det} 2$ mutants, which affect the expression of $C A B$ and $R B C S$ (the small subunit of RuBP carboxylase) to approximately the same extent, all three doc mutations are much more specific in derepressing the expression of $C A B$. The phenotypes of $d o c$ mutants suggest that morphological changes can be genetically separated from changes in $C A B$ gene expression. Moreover, the regulation of $C A B$ gene expression can be separated further from the regulation of $R B C S$ gene expression. Epistasis studies suggest that DOC1 and DET3 act downstream from DET1 on two separate branches in the phototransduction pathway. In contrast, DOC2 appears to act on a distinct pathway from DET1. Mutations in doc1, doc2, or doc3 also impair plant growth under short-day conditions.
\end{abstract}

[Key Words: Photomorphogenesis; light-regulated gene expression; chlorophyll $a / b$-binding protein; Arabidopsis mutants]

Received September 14, 1993; revised version accepted December 15, 1993.

Light plays a pivotal role in the development of plants. It regulates germination, leaf development, organelle morphology, photosynthesis, and gene expression /Chory 1991). One of the most important challenges in studying plant development is to understand the light-regulated signal transduction pathways that lead to these developmental responses.

The best-defined molecular event in light-induced responses is the expression of light-regulated genes, such as the genes encoding the chlorophyll $a / b$-binding proteins of photosystem II $(C A B)$, the small subunit of RuBP carboxylase $|R B C S|$, and chalcone synthase. Analyses of light-regulated expression of these genes have increased our understanding of the light signal transduction pathways. For example, deletion and mutational analyses of the promoters of these genes and in vitro protein-binding assays using promoter fragments have identified several cis-acting sequences and trans-acting factors with possi-

\footnotetext{
'Present address: Botanisches Institut der Ludwig-Maximilians-Universität, D-80638, München, Germany.

${ }^{2}$ Corresponding author.
}

ble functions in light-regulated transcription (for review, see Gilmartin et al. 1990; Li et al. 1993). Some common features can be found among the different cis-acting elements and also among the various trans-acting factors. For instance, two or more distinct cis-acting elements are required for the expression of a light-inducible promoter (Weisshaar et al. 1991) and the light-regulatory elements are redundant (e.g., Gilmartin et al. 1990). Also, in vitro DNA-binding activities of most nuclear factors (and the mRNA levels of those factors for which a cDNA has been isolated) are not light-regulated and are often found to be present in all tissues (Gilmartin et al. 1992; Perisic and Lam 1992; Schindler et al. 1992). Explanations for this lack of specificity are that post-translational modifications or some other protein factor, for example, a repressor in the dark or an activator in the light, are required for the proper function of these transacting factors. At present, the biological functions of these nuclear factors are not clear.

Genetics is an alternative tool for studying light-regulated gene expression, with the potential to identify genes on the basis of their functional importance. Mu- 
Li et al.

tants have been isolated that express light-induced genes at high levels in the dark, for example, several of the det (de-etiolated, Chory et al. 1989, 1991) and cop (constitutively photomorphogenic; Deng et al. 1991; Wei and Deng 1992) mutants of Arabidopsis thaliana and the lip (light-independent photomorphogenesis) mutant of pea (Frances et al. 1992). However, these mutants were isolated on the basis of their abnormal de-etiolated morphology in the dark, that is, they have opened cotyledons and short hypocotyls. Mutations identified this way are either pleiotropic and affect multiple downstream events or have no effect on light-regulated gene expression at all. For example, the det 1 and cop 1 mutants of Arabidopsis not only express light-regulated genes at relatively high levels but also make leaves and chloroplasts in the dark (Chory et al. 1989; Deng et al. 1991). Tissuespecific expression of several light-regulated genes in light-grown det 1 mutants is also altered /Chory and Peto 1990). Thus, DET1 and COP1 most likely regulate early steps in the light-regulated signal transduction pathways, and their effect on photosynthetic gene expression may be indirect. On the other hand, although the det 3 mutant and, to some extent cop 2 and cop 3 mutants have de-etiolated morphology in the dark, they exhibit a wildtype pattern of light-regulated gene expression (Cabrera y Poch et al. 1993; Hou et al. 1993).

We have designed a genetic screen that allows us to isolate mutations that specifically affect the expression of light-regulated genes in the absence of morphological changes. A stably transformed line of Arabidopsis was constructed that contained a transgene with two $C A B 3$ (also called $C A B 180$, Leutwiler et al. 1986) promoters fused to two different reporter genes (Chory et al. 1993). Seeds from transgenic plants homozygous for the transgene were mutagenized and used to screen for mutants that expressed both reporter genes at aberrantly high lev- els in the dark. We report here the characterization of several of the mutants isolated by use of this screen.

Genetic analyses indicated that most of the mutations were recessive and defined a minimum of three loci. Unlike previously isolated mutants, all of the mutants had normal etiolated morphology in the dark. At least two of the mutations specifically affected the accumulation of $C A B$, but not of $R B C S$, mRNAs in the dark. The existence of these mutants indicates that during dark-grown seedling development, $C A B$ gene expression can be separated from morphological changes. Moreover, regulation of $C A B$ expression can be genetically separated from $R B C S$ expression. A pathway positioning these genes in the light-regulated signal transduction chain is proposed based on epistasis studies of these mutants and the previously described $\operatorname{det} 1$ and $\operatorname{det} 3$ mutants.

\section{Results \\ Isolation of mutants using a dual promoter-reporter transgenic line}

We designed a reporter construct to identify mutants in the phototransduction pathway. The T-DNA construct, pOCA107, contained two full-length $C A B 3$ promoters fused either to the hygromycin phosphotransferase $(h p h)$ gene or to the Escherichia coli $\beta$-glucuronidase (uidA or GUS) gene (Fig. 1A). We transformed the Columbia ecotype of Arabidopsis with the construct and generated a number of transgenic lines. One of the resulting transgenic lines, pOCA107-2, was homozygous for a single insertion of the transgene on chromosome II and displayed proper light-regulated and tissue-specific expression of the CAB3-uidA transgene (Chory et al. 1993). To isolate mutants that had high $C A B 3$ promoter activity in the dark, $M_{2}$ progeny of ethylmethane sulfonate (EMS) or
Figure 1. Selection of doc mutants. $|A|$ Schematic representation of the pOCA107 T-DNA construct. (LB and RB) Left and right borders of the T-DNA, respectively. The kanamycin resistance gene (nptII) used for selection is driven by the constitutive cauliflower mosaic virus $35 \mathrm{~S}$ promoter (35S). One $C A B 3$ promoter was fused to the hygromycin phosphotransferase $(h p h)$ gene. The second $C A B 3$ promoter was fused to the $E$. coli $\beta$-glucuronidase (uidA) gene. All three reporter genes were fused to the nopaline synthase (nos) terminator. (B) Hygromycin resistance as measured by hypocotyl length. From the left are shown wild-type, two putative mutants, and the PHGl (positive control) line. Seedlings were grown for 7 days in the dark on media containing 20 $\mu \mathrm{g} / \mathrm{ml}$ of hygromycin B. (C) The eight $d o c$ mutants were grown on $25 \mu \mathrm{g} / \mathrm{ml}$ of hygromycin B for 7 days in the dark. From the left: Wild type, doc0-6, doc1, doc3, doc2-1, doc2-2, doc0-11, doc0-13, and doc0-19.
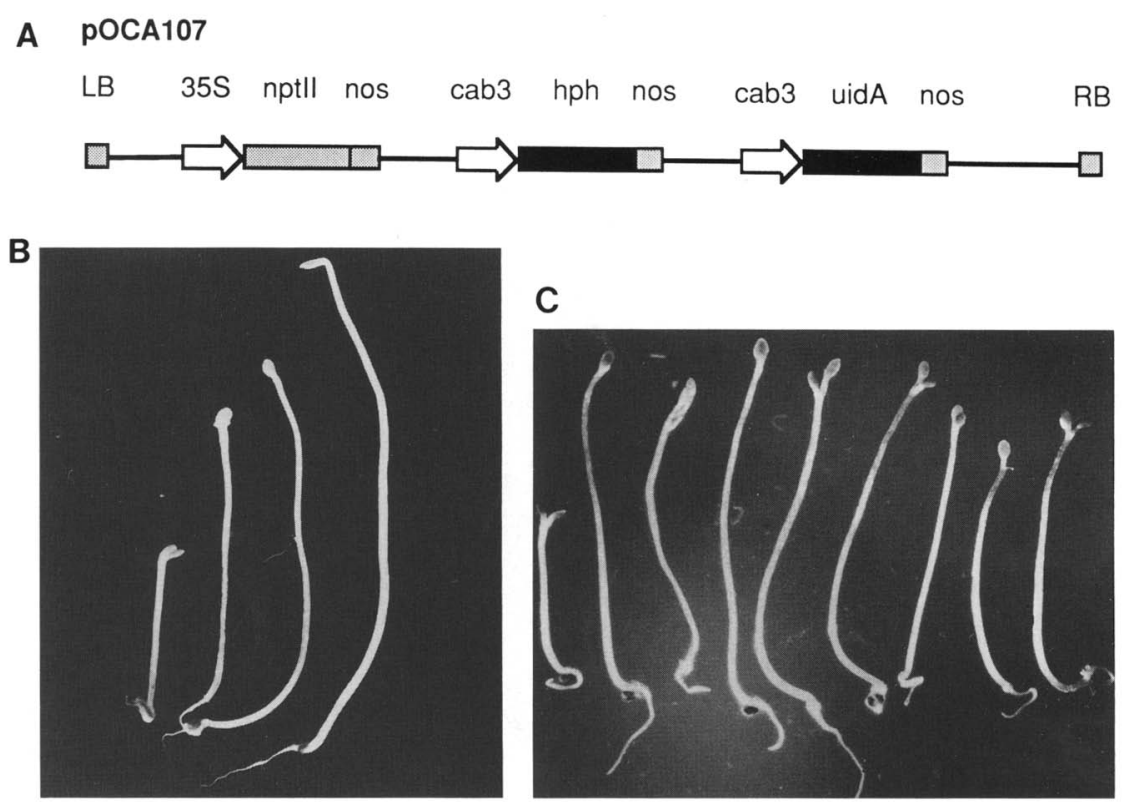
$\gamma$-ray mutagenized pOCA107-2 seeds were first screened in the dark for hygromycin resistance (i.e., for overexpression of the CAB3-hph transgene). A similar transgenic line, PHG1, in which the hph gene was fused to the phenylalanine ammonia-lyase (PAL) promoter (S. Ohl, B. Kraft, J. Chory, and C.J. Lamb, unpubl.), served as a positive control. Because the PAL promoter was highly expressed in the dark, the PHGl line was hygromycin resistant when grown in the dark on media containing hygromycin. It had a normal etiolated morphology with a hypocotyl length of $\sim 14 \mathrm{~mm}$ (Fig. 1B). In contrast, the unmutagenized pOCA107-2 line had a hypocotyl length of 1 to $4 \mathrm{~mm}$ (Fig. 1B). Hypocotyl length was thus used as an indicator for hygromycin resistance.

We selected 375 putative mutants with long hypocotyls on hygromycin media in the dark and allowed them to recover in the light and set seeds. To eliminate mutants that had only cis-acting mutations in the CAB3$h p h$ transgene, progeny from the putative mutants were screened further for the expression of the second transgene, $C A B 3-$ uidA, by assaying GUS activity of pooled dark-grown seedlings. Forty putative mutant lines also had higher specific GUS activities than the wild type. After rescreening for the expression of both reporter genes in the next few generations, eight putative mutant lines consistently showed longer hypocotyls on hygromycin-containing media (Fig. 1C) and had three- to sevenfold higher specific GUS activities (Table 1) than the wild type (pOCA107-2). These mutants most likely contained mutations that acted in trans to affect the expression of both $C A B 3$ promoters in the transgene. The putative mutants were thus designated $d o c$ for dark overexpression of $\underline{C} A B$. Several hygromycin-resistant mutants with a de-etiolated morphology (short hypocotyl and opened cotyledon) were also isolated during the screen. Genetic analyses indicated that they were allelic to the det 1 mutant $(\mathrm{H}$. Cabrera y Poch, L. Atlschmied, and J. Chory, unpubl.).

\section{Genetic analysis of doc mutants}

To determine the nature of the mutations, putative mutants were backcrossed to either the parent pOCA107-2

Table 1. Specific GUS activities of doc mutants

\begin{tabular}{lcc}
\hline Lines & $\begin{array}{l}\text { GUS activity } \\
\text { (pmole/mg per min) }\end{array}$ & $\begin{array}{l}\text { Ratio of } \\
\text { mutant/wild type }\end{array}$ \\
\hline pOCA 107 & $74.3 \pm 0.0$ & 1.0 \\
doc1 & $500.9 \pm 2.5$ & 6.7 \\
doc2-1 & $526.9 \pm 20.0$ & 7.1 \\
doc2-2 & $226.8 \pm 13.3$ & 3.1 \\
doc3 & $277.0 \pm 2.4$ & 3.7 \\
doc0-6 & $249.7 \pm 0.0$ & 3.4 \\
doc0-11 & $270.5 \pm 18.9$ & 3.6 \\
doc0-13 & $377.0 \pm 3.3$ & 5.1 \\
doc0-19 & $485.2 \pm 17.0$ & 6.5 \\
\hline
\end{tabular}

GUS activity was assayed on seedlings grown for 6 days in the dark. line or to the wild-type Columbia ecotype. If the mutations were recessive, the $F_{1}$ progeny from the backcross should be hygromycin sensitive with short hypocotyls, that is, wild type in appearance. As shown in Table 2, most of the mutant lines arose from single gene recessive mutations, except doc0-6. doc0-6 was a dominant mutation as indicated both by the backcross and by complementation crosses with other mutants (Table 3). We have not finished the studies with $d o c 0-19$. doc3 and doc0-13 were most likely single gene recessive mutations, even though the segregation ratios of the $F_{2}$ progeny fitted better with $3: 1$ instead of the expected 13:3 (see Table 2 footnote). This might indicate that the mutations were linked to the pOCA107-2 transgene or simply that we encountered difficulty when scoring the mutants.

To determine how many complementation groups these new mutations defined, the mutants were crossed to one another, and the $F_{1}$ progeny were tested for resistance to hygromycin. The results indicated that there were two alleles of $d o c 2$, and $d o c 1$ appeared to complement doc2-1, doc2-2, and doc3 (Table 3). We confirmed this by mapping docl, doc2-1, and doc3. These three mutants were crossed to either Landsberg or Niederzenz ecotypes, and the DNAs from the $F_{2}$ progeny were analyzed using codominant ecotype-specific PCR-based markers (Konieczny and Ausubel 1993). The DOC1 locus was mapped to the upper portion of chromosome III between the markers GapC and myb4. DOCl was $\sim 3$ centimorgans (cM) from $G a p C$ and $2 \mathrm{cM}$ from myb4. The $D O C 2$ locus was positioned between the markers $D F R$ (tt3) and ASAl on chromosome $\mathrm{V}, \sim 34 \mathrm{cM}$ from DFR. The DOC3 locus was mapped to the lower portion of chromosome I between the markers GapB and $A D H$ $(\sim 26 \mathrm{cM}$ from GapB and $\sim 31 \mathrm{cM}$ from $A D H)$. The dominant mutation, doc0-6, was mapped to chromosome II $\sim 23 \mathrm{cM}$ from the pOCA107-2 transgene. Thus, we have identified at least four loci that affected the expression of the two $C A B 3$ promoters in the transgene, with one allele of docl, doc3, and doc0-6 and two alleles of doc2.

doc mutations affect the accumulation of endogenous $\mathrm{CAB} m R N A s$ and define a new branch in the signal transduction pathway

Because the mutations in the doc mutants increased the expression of both $C A B 3$ promoters in the transgene, it was likely that the endogenous $C A B$ promoters were also affected. Figure 2 shows the result of an RNA gel blot hybridized with a $C A B$ gene probe that detects mRNAs from all members in the photosystem II type I $C A B$ gene family. All of the mutants, except doc0-6, had elevated endogenous $C A B$ transcript levels in the dark when compared to the wild type. The extent of derepression varied with each allele. On the basis of three to four separate experiments and densitometer scans, docl had $\sim 6.2$-fold elevation of $C A B$ mRNAs over the wild type, and doc2-1 and doc3 had $\sim 3.5$ - and 3.2-fold elevation, respectively. The remaining mutants had less than two- 
Table 2. Results of crosses with doc mutants

\begin{tabular}{|c|c|c|c|c|c|}
\hline \multirow[b]{2}{*}{ Cross } & \multirow[b]{2}{*}{$F_{1}$} & \multicolumn{4}{|c|}{$\mathrm{F}_{2}$} \\
\hline & & $\begin{array}{l}\text { wild type } \\
\text { (hygromycins }^{\text {(h) }}\end{array}$ & $\begin{array}{l}\text { mutant } \\
\text { (hygromycin }{ }^{\mathrm{r}} \text { ) }\end{array}$ & $\begin{array}{l}\text { ratio of } \\
\text { wild type/mutant }\end{array}$ & $x^{2}$ \\
\hline Co $\times$ doc1 & WT & 54 & $16^{a}$ & 3.38 & $0.17^{b}$ \\
\hline doc2-1 $\times$ pOCA 107 & WT & 163 & 62 & 2.63 & $0.78^{\mathrm{b}}$ \\
\hline pOCA107 $\times$ doc $2-2$ & WT & 447 & 154 & 2.90 & $0.12^{\mathrm{b}}$ \\
\hline Co $\times$ doc 3 & WT & 184 & 61 & 3.02 & $0.001^{b}$ \\
\hline Co $\times$ doc0-6 & mutant $\mathrm{t}^{\mathrm{c}}$ & 222 & 406 & 0.55 & \\
\hline Co $\times \operatorname{doc} 0-11$ & N.D. & 95 & 28 & 3.39 & $1.3^{\mathrm{d}}$ \\
\hline Co $\times \operatorname{doc} 0-13$ & WT & 139 & 50 & 2.78 & $0.21^{\mathrm{b}}$ \\
\hline
\end{tabular}

Each cross is indicated as female parent $\times$ male parent. Seedlings were scored for hygromycin resistance by measuring hypocotyl lengths after growth for 7 days in the dark on media containing $25-40 \mu \mathrm{g} / \mathrm{ml}$ of hygromycin $\mathrm{B}$. If the mutant phenotype arises from a single gene recessive mutation, the $F_{1}$ progeny from the cross should be hygromycin sensitive (short hypocotyls), i.e., wild type (WT). $F_{2}$ progeny should show a segregation ratio of $3: 1$ wild type/mutant if the mutant was crossed to pOCA107, or a ratio of $13: 3$ wild type/mutant if the mutant was crossed to Columbia $(\mathrm{Co})$, as one-fourth of the $\mathrm{F}_{2}$ mutant seedlings would not have the pOCA107 transgene, which is necessary to confer hygromycin resistance. (N.D.) Not determined.

The mutant was scored with the phenotype of reduced apical dominance.

bThe $\chi^{2}$ value is given for the ratio of $3: 1$ (wild type/mutant).

${ }^{c}$ The $F_{1}$ seedlings showed a mixture of hygromycin-sensitive and -resistant plants, indicating that the mutant parent could be heterozygous for the mutation, or the hygromycin-sensitive seedlings resulted from selfing of the Columbia parent. The hygromycinresistant $F_{1}$ plants were used to generate the $F_{2}$ progeny.

${ }^{\mathrm{d}}$ The $\chi^{2}$ value is given for the ratio of $13: 3$ (wild type/mutant).

fold increases of $C A B$ mRNA over the wild type, and we did not study them further. Surprisingly, doc0-6 did not have elevated $C A B$ mRNA accumulation, despite the observations that the seedlings were hygromycin resistant, had a higher specific GUS activity than the wild type, and the mutation mapped $23 \mathrm{cM}$ away from the transgene.

The expression of another light-regulated nuclear gene, $R B C S$, was also analyzed (Fig. 2). For most mutants the accumulation of $R B C S$ mRNA was not as high as the $C A B$ mRNA. In particular, in doc2 and doc3 mutants $R B C S$ transcript levels were approximately the same as in the wild type (a normalized mutant/wild type ratio of $1.5 \pm 0.5$ and $1.4 \pm 0.2$ respectively, on the basis of four experiments). In the docl mutant, RBCS mRNA levels

Table 3. Complementation crosses among doc mutants

\begin{tabular}{|c|c|c|}
\hline \multirow[b]{2}{*}{ Cross } & \multicolumn{2}{|c|}{$F_{1}$} \\
\hline & $\begin{array}{l}\text { mutant } \\
\text { (hygromycin }{ }^{\mathrm{T}} \text { ) }\end{array}$ & $\begin{array}{l}\text { wild type } \\
\text { (hygromycin }\end{array}$ \\
\hline $\operatorname{doc} 2-1 \times \operatorname{doc} 1$ & 0 & 6 \\
\hline$d o c 1 \times d o c 3$ & 0 & 4 \\
\hline doc $3 \times d o c 1$ & 0 & 6 \\
\hline doc2-1 $\times$ doc $2-2$ & 32 & 1 \\
\hline docl $\times$ doc0-13 & 0 & 17 \\
\hline doc $1 \times$ doc $2-2$ & 0 & 4 \\
\hline doc $0-19 \times$ doc0-13 & 0 & 3 \\
\hline doc $0-6 \times d o c l$ & 13 & 0 \\
\hline $\operatorname{doc} 0-6 \times \operatorname{doc} 2$ & 4 & 0 \\
\hline doc $0-6 \times$ doc 3 & 16 & 0 \\
\hline doc $0-13 \times d o c 0-6$ & 24 & 0 \\
\hline
\end{tabular}

All conditions are the same as described in Table 2. were two- to fivefold higher than the wild-type levels. These results indicated that mutations in DOC2 and $D O C 3$ specifically affected the dark expression of $C A B$ but not $R B C S$ genes. $D O C 1$ had a greater effect on $C A B$ than $R B C S$ mRNA accumulation. In contrast to other mutations like $\operatorname{det} 1$ and $\operatorname{det} 2$ (Chory et al. 1989, 1991), which affect the expression of $C A B$ and $R B C S$ to approximately the same extent, DOC2 and DOC 3 defined a new branch in the signal transduction pathway that was specific for the regulation of $C A B$. On the basis of the above data, docl, doc2-1, and doc3 were chosen for further analyses because they had the highest elevation of $C A B$ transcripts in the dark.

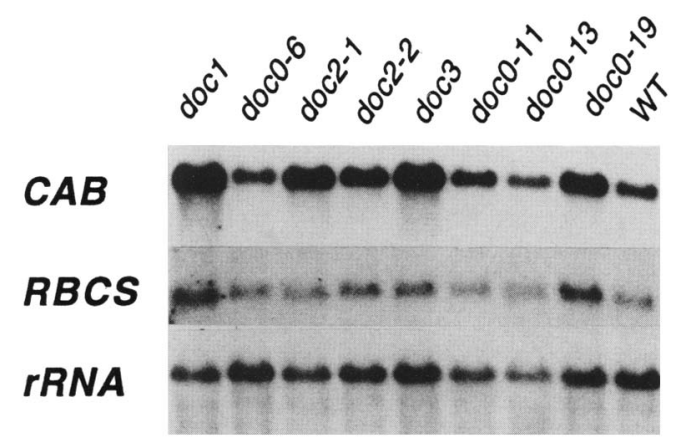

Figure 2. Accumulation of $\mathrm{mRNAs}$ for $C A B$ and $R B C S$ in darkgrown doc mutants as determined by an RNA gel blot. Seedlings were grown for 4 days in the dark on synthetic media. Total RNA $(5 \mu \mathrm{g})$ from individual mutants (top) was loaded per lane. The $C A B$ and $R B C S$ probes used detect all members in the two gene families. The RNA load in each lane was normalized by probing for rRNA. (WT) Wild type. 
$\mathrm{CAB} 1$ transcripts are also increased in the doc mutants

Arabidopsis has at least five members in the photosystem II type I CAB gene family (Leutwiler et al. 1986; McGrath et al. 1992). For the three promoters for which expression has been characterized $\mid C A B 1, C A B 2$, and $C A B 3$, also called $C A B 140, C A B 165$, and $C A B 180$, respectively; Leutwiler et al. 1986), it has been shown that the three transcripts are differentially expressed both in the light and in the dark (Sun and Tobin 1990; Brusslan and Tobin 1992). The sequence of the $C A B 1$ promoter is less homologous to that of the $C A B 2$ and $C A B 3$ promoters, which are very similar to each other (Leutwiler et al. 1986). $C A B 1$ transcripts also constitute the majority of $C A B$ messages in wild-type dark-grown seedlings (Brusslan and Tobin 1992). Because our mutants were isolated on the basis of increased hygromycin resistance and GUS activity (Table 1), which were reflections of only CAB3 promoter activity, and the RNA gel blots as shown in Figure 2 could not distinguish among the various $C A B$ transcripts, we sought to investigate whether $C A B 1$ message levels were also affected in the doc mutants. Figure 3 shows the result of an RNase protection experiment with a $C A B 1$-specific probe (Brusslan and Tobin 1992). The levels of $C A B 1$ transcripts in the three $d o c$ mutants were compared with a ubiquitin gene (UBQ3), which was used as a constitutive control (Norris et al. 1993). The result showed that $C A B 1$ transcripts were elevated in the three doc mutants, indicating that the three doc mutations derepressed both $C A B 3$ and $C A B 1$ expression in the dark.

\section{Phenotypes of doc mutants}

All of the doc mutants had normal etiolated morphology when grown in the dark (Fig. 1C; see also Fig. 5, below), indicating that unlike all of the other photomorphogenic mutants isolated to date, these mutations affected the expression of downstream photosynthetic genes in the absence of obvious morphological changes. The simplest

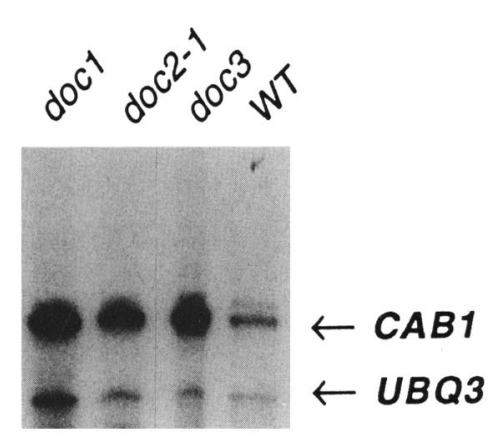

Figure 3. $C A B 1$ transcript accumulation in doc mutants as determined by RNase protection analysis. Seedling were grown for 4 days in the dark on synthetic media. Total RNA $(3.5 \mu \mathrm{g})$ from individual mutants (top) was loaded per lane. Levels of $U B Q 3$ RNA were also determined and used to normalized the amount of RNA in each lane. (WT) Wild type. model to explain our mutants is that they are defective in the normal light/dark expression of $C A B$. Brusslan and Tobin (1992) have shown recently that dark-grown Arabidopsis seedlings exhibit a developmental regulation of $C A B$ and $R B C S$ gene expression that is independent of light. In their studies, the expression of $C A B$ mRNA transiently peaks at 3 days postgermination and then gradually drops back to basal level.

We analyzed the timing of $C A B$ gene expression in dark-grown doc mutants. In the three doc mutants, both $C A B$ mRNA levels and specific GUS activities from the $C A B 3$ promoter were elevated to the same extent over the levels in wild-type seedlings of the same age throughout the entire time course studied (2-8 days; data not shown). This indicated that the developmental timing of $C A B$ expression was not altered in dark-grown doc mutants. Moreover, the level of $C A B$ mRNA in light-grown doc mutants was not significantly different from the wild type (data not shown). Finally, cell-type-specific expression of $C A B$ genes in either light- or dark-grown $d o c$ mutants, as analyzed by histochemical staining of GUS activity, was not altered (data not shown). These results suggested that mutations in the three $D O C$ genes were specific for a pathway(s) that functioned to repress $C A B$ gene expression in etiolated seedlings.

Of all the mutants, only docl had a clear growth phenotype when grown in the light. Under long-day growth conditions ( $18 \mathrm{hr}$ light $/ 6 \mathrm{hr}$ dark), mature docl plants had reduced apical dominance (seven to nine inflorescence stems; data not shown) compared with the wild type (one to three inflorescence stems). doc1 was also shorter than the wild type. In contrast, doc2 and $d o c 3$ were almost indistinguishable from the wild type when grown under long-day conditions (Fig. 4A). However, when grown under short-day conditions $(9 \mathrm{hr}$ light $/ 15 \mathrm{hr}$ dark), all three $d o c$ mutants were significantly smaller than the wild type and had less than half of the fresh weight of the wild type (Fig. 4B). Thus, mutations that affected transcription of $C A B$ promoters in the dark also seemed to have an effect on plant growth when the day length was short.

\section{det-doc double mutant studies}

It has been shown that mutations in the DETl gene affect several aspects of seedling development in the dark. For example, dark-grown det 1 mutant plants develop as light-grown plants with respect to morphology and the expression of several light-regulated genes (Chory et al. 1989|. On the other hand, mutations in the DET3 gene result in seedlings with a de-etiolated morphology similar to det 1 , but the expression of light-regulated genes is not derepressed, resembling the wild type (Cabrera y Poch et al. 1993). Double mutant studies suggest that DET3 lies downstream from DETl on a branch of the pathway that affects only seedling morphology. We sought to study the epistatic relations and possible interactions of $\operatorname{det} 1$ and $\operatorname{det} 3$ with $d o c 1$ and $\operatorname{doc} 2$ by generating double mutants containing mutations in both groups of genes. 


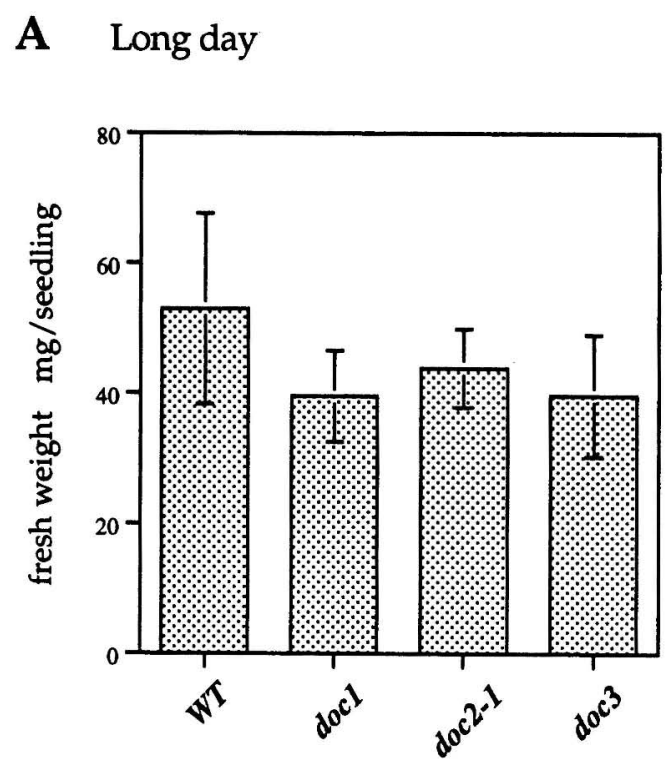

B Short day

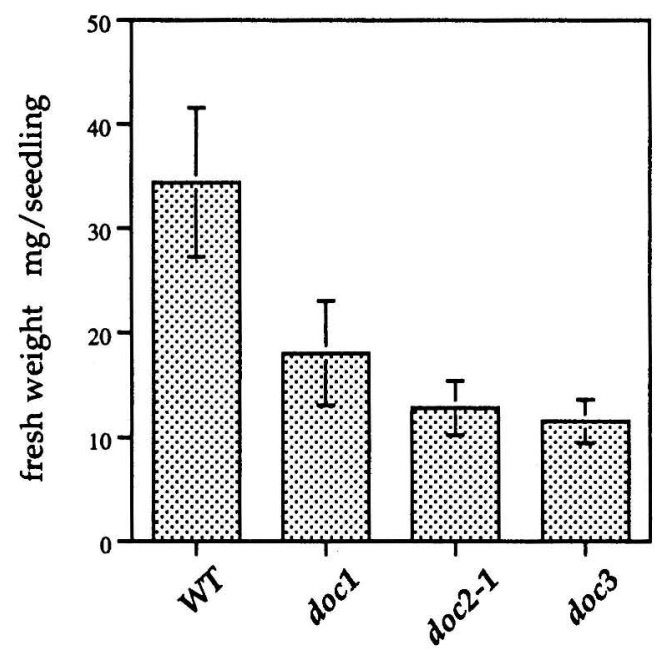

Figure 4. Phenotypes of doc mutants when grown in short days. The fresh weights of $d o c$ mutants were reduced. Plants were grown for 34 days on a synthetic medium in an 18-hr light/6-hr dark long-day cycle $(A)$, or a 9 -hr light/15-hr dark short-day cycle $(B)$. At least five plants were measured for each mutant and the wild type (WT).

Seedlings homozygous for both the detl and docl mutations (see Materials and methods) had a morphology identical to the detl mutant both in the light (data not shown) and in the dark (Fig. 5A). Gene expression changes in the double mutant were analyzed by assaying specific GUS activity of dark-grown doubly mutant seedlings. As shown in Figure 6A, GUS activity of darkgrown det1-docl was comparable to that of the $\operatorname{det} 1$ parent. This indicated that the det 1-docl double mutant had the phenotype of detl with respect to both $C A B$ gene expression and morphological changes, suggesting that
DET1 and DOC1 were on the same signal transduction pathway.

In contrast, seedlings homozygous for det 1 and doc 2 mutations had the additive phenotype of $\operatorname{det} 1$ and $\operatorname{doc} 2$. Whereas the double mutant had the morphology of det 1 in the dark (data not shown), the specific GUS activity of the dark-grown double mutant was equal to the activity of det 1 plus doc2 (Fig. 6B). This suggested that the two mutations had independent effects on transcription of the $C A B 3$ promoter and might act on different signal transduction pathways.

We have also generated the double mutants, det3docl and det3-doc2. Both double mutants had the appearance of $\operatorname{det} 3$ in the dark $(\operatorname{det} 3-\operatorname{doc} 2$, Fig. $5 \mathrm{~B}$; det 3 docl, data not shown). We did not have a det3 line containing the pOCA107 transgene; however, dark-grown $\operatorname{det} 3$ has $C A B$ transcript levels that are similarly low to the wild type (Cabrera y Poch et al. 1993). Therefore, we felt it was valid to compare the specific GUS activity of dark-grown double mutants to that of the wild type. As shown in Figure 6C, GUS activities of the dark-grown double mutants were elevated as in the doc mutants, although the degree of elevation was slightly lower than in the doc single mutants. These results suggested that the two double mutants had the additive phenotypes of $\operatorname{det} 3$ and the $d o c$ mutants, suggesting that the $\operatorname{det} 3 \mathrm{mu}$ tation and the two doc mutations affected two separate pathways or two different branches of a single pathway.

\section{Discussion}

We have identified three new Arabidopsis loci (docl, $d o c 2$, and $d o c 3$ ), mutations in which allow derepression of $C A B$ expression in dark-grown seedlings in the absence of morphological changes. The phenotypes of these mutants and epistasis studies suggest that these new genes define downstream branches of the light-regulated signal transduction pathways. $\operatorname{doc} 2$ and $\operatorname{doc} 3 \mathrm{mu}-$ tations specifically affect the expression of $C A B$ genes with little effect on $R B C S$ gene expression. Though $C A B$ and $R B C S$ genes have been shown to be differentially regulated by light fluence (Kaufman et al. 1984), this is the first genetic evidence that the light-regulated expression of $C A B$ and $R B C S$ genes can be separated. Because the mutations are recessive and affect the expression of multiple genes, these three loci may encode negative regulators or affect the activity of negative factors that repress the expression of $C A B$ in the dark.

By a number of criteria, the doc mutations are specific for a pathway(s) that functions to repress $C A B$ gene expression in etiolated seedlings. First, the levels of $C A B$ mRNAs are not derepressed in light-grown doc seedlings. In addition, doc mutants maintain the normal celltype-specific expression of $C A B$ in both light- and darkgrown seedlings. Finally, the light-independent developmental expression of $C A B$ (Brusslan and Tobin 1992) in etiolated seedlings is not altered in doc mutants. However, the three doc mutations do have some deleterious effects on plants grown in short-day conditions. docl, $d o c 2-1$, and $d o c 3$ all had severely reduced fresh weights 
A

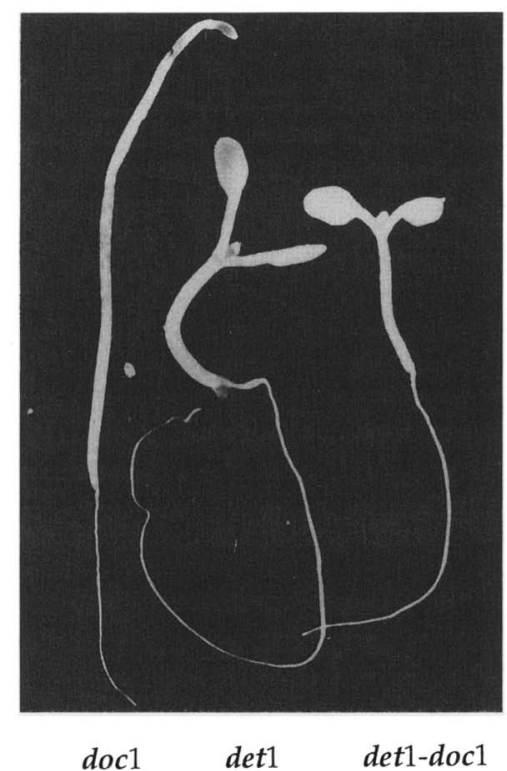

B

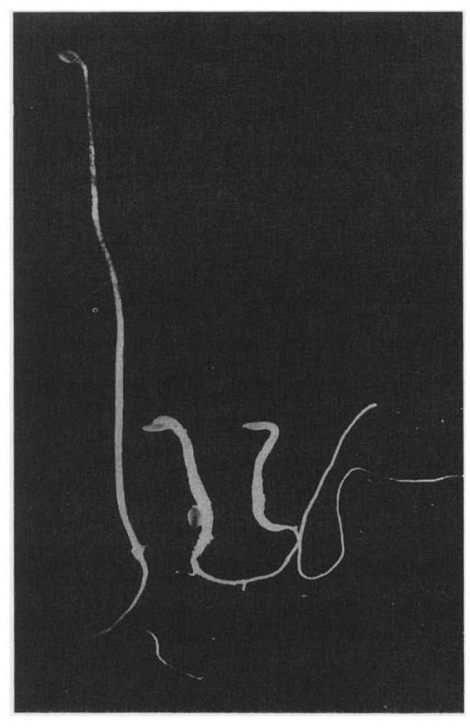

$\operatorname{doc} 2 \operatorname{det} 3 \operatorname{det} 3-\operatorname{doc} 2$
Figure 5. Dark-grown phenotypes of detl$\operatorname{doc} 1|A|$ and $\operatorname{det} 3-\operatorname{doc} 2(B)$ double mutants. Seedling were grown for 7 days in the dark on synthetic media. when grown under short-day conditions (Fig. 4). One explanation for this observation is that doc mutations result in slower repression of $C A B$ gene transcription in the dark. Longer nights would mean more energy is wasted on $C A B$ expression in the dark and thus result in impaired growth. An alternative explanation is that the full activation of $C A B$ gene expression in the light might require a total inactivation of some repressors that accumulate to down-regulate these genes during the night. Mutations in the DOC genes could result in partial or slower inactivation of the repressors in the light. Thus, longer day length might be required for the mutants to assimilate enough photosynthates to reach wild-type growth levels.

When etiolated seedlings are transferred to the light, a number of developmental and gene expression changes occur. The expression of light-regulated genes, for instance, is induced by $\sim 30$ - to 50 -fold over the basal dark levels (Chory et al. 1989). Numerous positively acting cis regulatory elements have been identified in the promoters of light-regulated genes, such as $C A B$ and $R B C S$ (for review, see Gilmartin et al. 1990; Li et al. 1993). These light-regulatory elements are redundant; with the exception of the G-box element (Donald and Cashmore 1990;
A $\operatorname{det} 1-\operatorname{doc} 1$

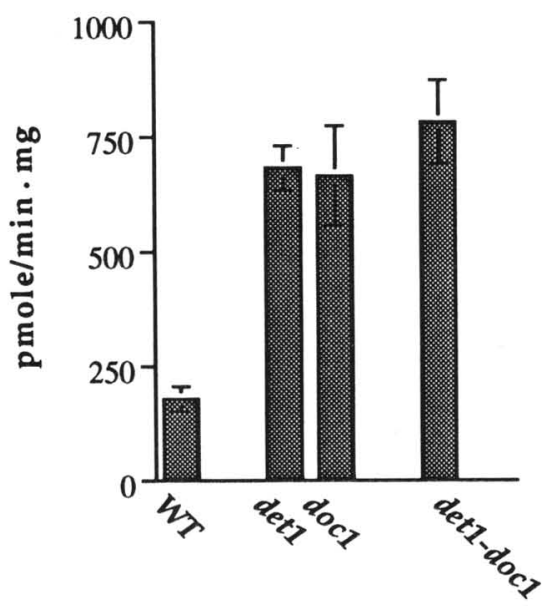

B $\operatorname{det1-doc2}$

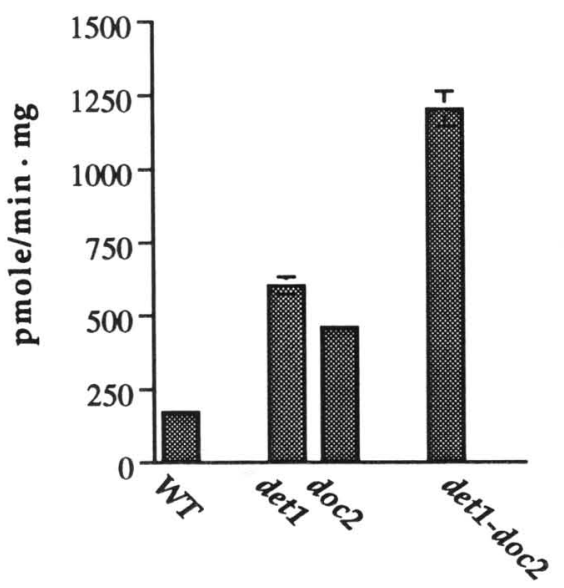

C $\operatorname{det} 3-\operatorname{doc} 1 \& \operatorname{det} 3-\operatorname{doc} 2$

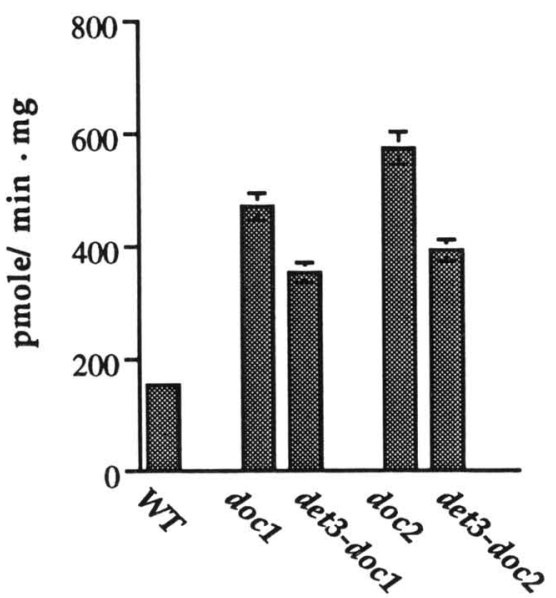

Figure 6. Specific GUS activities of dark-grown $\operatorname{det} 1-\operatorname{doc} 1|A|, \operatorname{det} 1-\operatorname{doc} 2(B), \operatorname{det} 3-\operatorname{doc} 1$ and $\operatorname{det} 3-\operatorname{doc} 2(C)$ double mutants. Seedlings were grown for 6 days in the dark. For several samples the standard errors (on the basis of at least four data points from two independent experiments) are too small to be seen in the scale plotted. 
S. Kay, pers. comm.|, mutation of an individual element results in no or small changes in promoter activity (three- to eightfold decreases where quantitative data are available; Castresana et al. 1988; Gidoni et al. 1989; Schulze-Lefert et al. 1989). In contrast, there are only a limited number of studies that address negatively acting cis-acting elements in these promoters. For instance, a mutation in an upstream negative regulatory element in the Nicotiana plumbaginifolia $C A B E$ promoter results in a fourfold increase of expression of this promoter (Castresana et al. 1988). A mutation in a negative regulatory element in the Arabidopsis CAB2 promoter causes three- to fourfold increases in $C A B 2$ expression in etiolated seedlings (S. Kay, pers. comm.). In Lemna, a number of genes have been identified that are negatively regulated by light. Deletion of the cis sequence responsible for increased expression in the dark in one of the promoters results in a 3.5-fold decrease in accumulation of the mRNA (Okubara et al. 1993). The level of derepression of the $C A B 3$ promoter in the doc mutants may reflect the somewhat modular nature of $C A B$ promoters. We observed a derepression of three- to sevenfold in $C A B$ promoter activity and mRNA accumulation in the three doc mutants described here. This is directly correlated with the levels of derepression associated with $C A B$ promoters when cis-acting negative regulatory elements are individually mutated. Experiments are in progress to attempt to correlate a specific $d o c$ mutation with a defined cis-acting regulatory element. Further genetic and biochemical studies may help to elucidate whether the $D O C$ gene products act additively or synergistically to affect the activity of the $C A B$ promoters.

$C A B$ and $R B C S$ represent the two best-studied lightregulated genes in plants. Proteins encoded by these two gene families are central elements in photosynthesis. Expression of both gene families is under phytochrome and blue light control (Thompson and White 1991), and similar promoter sequences have been identified in both promoters (Gilmartin et al. 1990). These data imply that expression of these two genes may be coordinately regulated by light. However, there is also indirect evidence suggesting that these two genes are differentially regulated. For example, the expression of $C A B$ genes is more sensitive to photo-oxidative damage (Susek and Chory 1992). High levels of cytosolic carbohydrates result in a much greater suppression of $R B C S$ gene expression than of $C A B$ gene expression (Krapp et al. 1993). $C A B$ is induced by very low fluences of light, whereas $R B C S$ requires higher fluences (Kaufman et al. 1984). doc2 and doc3 derepress the expression of $C A B$ in the dark with little or no effect on the expression of $R B C S$. It is reasonable to postulate that $C A B$ and $R B C S$ genes have different requirements for and sensitivities to light stimulation and metabolic balance in plants, considering their roles in light-harvesting and carbon fixation, respectively. Thus, separate mechanisms may have evolved to repress $C A B$ and $R B C S$ expression in the dark. It is possible that DOC2 and DOC3 respond to some environmental conditions that require the modulation of $C A B$, but not RBCS, expression. DOC2 and DOC3 then regu- late the activities of $C A B$ promoters by interacting with unique sequences in the promoters or by modifying the activities of some $C A B$-specific transcription factors. Alternatively, it is possible that the $d o c 2$ and $d o c 3$ mutations are leaky alleles of a group of transcription factors with different affinities for the $C A B$ and $R B C S$ promoters.

It has been shown that the three $C A B$ promoters of Arabidopsis (CAB1,CAB2, and $C A B 3)$ are differentially regulated (Karlin-Neumann et al. 1988; Sun and Tobin 1990). In the Columbia ecotype, $C A B 1$ is the major transcript in the dark and the $C A B 1$ promoter is also much more responsive to red light stimulation than the other two $C A B$ promoters (Karlin-Neumann et al. 1988; Brusslan and Tobin 1992). In vitro binding assays also indicate that some protein factors that bind to the $C A B 1$ promoter do not bind to the $C A B 2$ promoter (S. Kay, pers. comm.). However, both $C A B 1$ and $C A B 3$ expression are derepressed by the mutations in the $d o c \mathrm{mu}$ tants that we have analyzed. This indicates that the three $C A B$ promoters still share some common regulators in the dark. The three $D O C$ loci may encode proteins that act directly on the three promoters, or they may encode proteins that modify the activities of transcription factors that regulate the expression of some or all $C A B$ promoters. Interestingly, although $\operatorname{doc} 3$ has the lowest level of $C A B 3$ promoter activity, it has the highest level of $C A B 1$ transcripts of the three doc mutants. This could indicate that different regulatory factors may have variable affinities for different promoters. Modulation of the availability of these factors, for example, by temporal expression or post-translational protein modifications, may result in differential expression of the various $C A B$ promoters.

doc0-6 is an unusual dominant mutation, mapping 23 $\mathrm{cM}$ from the transgenes. This mutation affects the expression of the reporter genes but not the endogenous $C A B$ genes. This suggests that the $d o c 0-6$ mutation may have some distal cis effect on the expression of the transgenes. This is different from a recently reported Arabidopsis mutation that resulted in lower $C A B$ mRNA levels (Brusslan et al. 1993). This mutation mapped to the transgene itself, causing a distal effect on the expression of the endogenous $C A B$ genes. However, we cannot exclude the possibility that the doc0-6 mutation has some small but specific effect on the expression of endogenous $C A B 2$ and/or $C A B 3$ promoters. Because the $C A B 1$ transcript is the major transcript in the dark (Brusslan and Tobin 1992), a small increase in $C A B 2$ or CAB3 transcripts may not be detected by RNA gel blots.

On the basis of our epistasis studies, we propose a model that incorporates the $D O C$ gene products in to the proposed phototransduction pathway (Fig. 7). Our previous studies have indicated that DET1 and DET2 act downstream from both phytochrome and a blue-light photoreceptor and most likely on separate branches of the signal transduction pathways (Chory 1992). Moreover, det 1 is epistatic to det3, suggesting that DET1 acts upstream from DET3, whereas the $\operatorname{det} 2-\operatorname{det} 3$ double mutant shows the phenotype of both parents /Cabrera y 


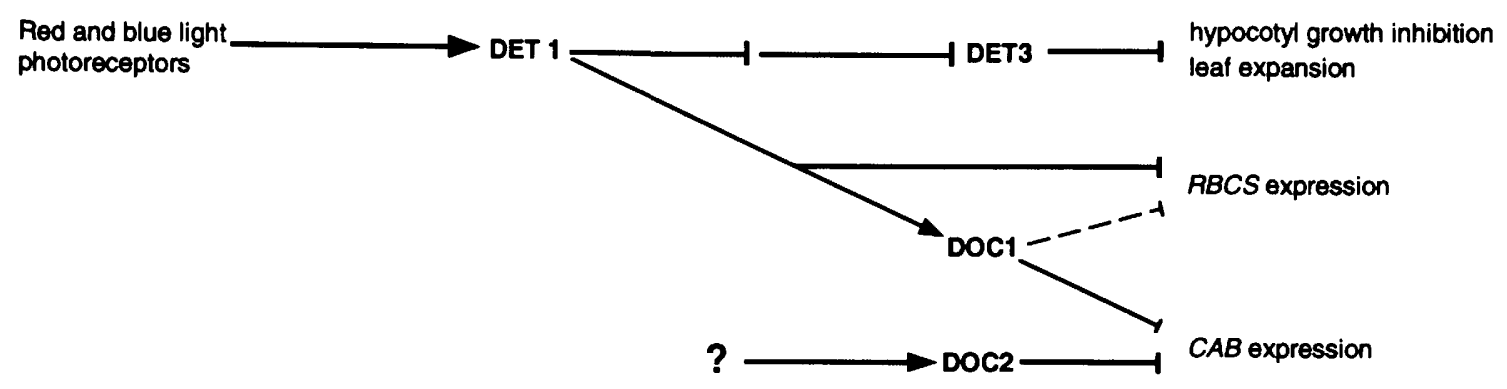

Figure 7. Model for the hierarchical relationships among DET and DOC gene products in the light-regulated signal transduction pathway. DETl is downstream from the red and blue light photoreceptors and negatively controls multiple branches in the pathway hereafter as indicated (Chory 1992). DET3 lies on a branch of the pathway that leads to changes in morphology (Cabrera y Poch et al. 1993), and the $D O C 1$ gene product lies on the $C A B$ gene expression branch of the same pathway and may also have a small effect on the expression of $R B C S$ genes. DOC2 appears to specifically repress the expression of $C A B$ but not $R B C S$. Its effect on the repression of $C A B$ mRNA accumulation is independent of DET1; thus, it may lie on a separate signal transduction pathway from DET1. The model is formal and makes no prediction as to the precise molecular nature of the proposed interactions among genes or gene products. $(\rightarrow \mid$ Positive regulation; $(-1)$ negative regulation. The broken line indicates that the docl mutation has a smaller effect on $R B C S$ gene expression than on $C A B$ gene expression.

Poch et al. 1993). Therefore, it is hypothesized that DET2 acts on a separate pathway from DET1 and DET3 (Cabrera y Poch et al. 1993). In the studies presented here, the detl-docl double mutant has the morphology and gene expression pattern of detl, suggesting that DOCl and DETl are in the same signal transduction pathway. This is consistent with the observation that light-grown docl also has reduced apical dominance as does det 1 . Because the docl mutant displays only a subset of the phenotypes of the det 1 mutant, we hypothesize that DOCl is downstream from DETl. In contrast, doc2 and det l appear to have independent effects on the expression of $C A B$ because in the det $1-\operatorname{doc} 2$ double mutant, $C A B 3$ promoter activity is derepressed to a level that is equal to the addition of the two parental mutants. This suggests that DOC2 is not directly downstream from DET1. It is possible that DOC2 is under the control of another upstream regulator like DET2 in the lightregulated signal transduction pathways. Alternatively, the regulation of the $C A B$ promoters by $D O C 2$ may respond to signals other than light, for example, the level of carbon source in the growth media (Brusslan and Tobin 1992; Sheen 1990) or localized hormone concentrations (Chory et al. 1994). Finally, the det3-docl and det3-doc2 double mutant each have the nearly additive phenotypes of the two parents, suggesting that DET3 and these two DOC gene products act on different branches of the pathways. However, the double mutant phenotypes are not perfectly additive: The GUS activities of the double mutants are slightly reduced when compared with the parental $d o c$ mutants. It is possible that the $\operatorname{det} 3$ mutation may have some negative effect on $C A B$ gene expression in the dark.

The doc mutants reported here have helped define two branches in the light-regulated signal transduction pathway. The three $D O C$ gene products separated the pathway of $C A B$ expression from the pathway of hypocotyl growth and leaf expansion and also divided the pathway of $C A B$ expression from $R B C S$ expression. With the iso- lation of these mutants, a more complete picture of the light-regulated signal transduction pathways is beginning to emerge. In the future, consolidating genetic with molecular and biochemical approaches will help in revealing the molecular mechanisms controlling light-regulated gene expression in seedling development.

\section{Materials and methods}

\section{Plant materials and growth conditions}

In all of the experiments described here, the wild type is the unmutagenized pOCA107-2 line in the Columbia background. Plants grown in the light on a synthetic medium (Murashige and Skoog salt mix, Gamborg's vitamin mix, and 2\% sucrose) were maintained in growth chambers with a temperature of $21^{\circ} \mathrm{C}$ and a light intensity of $350 \mu \mathrm{E} / \mathrm{m}^{2}$ per sec. Plants grown in the greenhouse on soil were maintained as described (Somerville and Ogren 1982). For dark-grown seedlings, seeds plated on the synthetic medium were cold-treated for $48 \mathrm{hr}$ in the dark and then transferred to the light for $24 \mathrm{hr}$. The end of this 24-hr light treatment was considered to be the beginning of the darkgrowth period. Plates were then wrapped in two layers of aluminum foil and kept in a dark growth chamber in a dark room for the amount of time indicated in each experiment.

\section{Mutagenesis and mutant isolation}

pOCA107-2 seeds were mutagenized with EMS (Chory et al. 1989 ) or $\gamma$-ray $(30 \mathrm{krad}$ ). A total of 100,000 EMS-mutagenized $\mathrm{M}_{2}$ seeds from 200 pools were screened for hygromycin resistance by growing the seedlings in the dark on a medium containing $40 \mu \mathrm{g} / \mathrm{ml}$ of hygromycin B for 7.5 days. Seedlings with noticeably longer hypocotyls than the wild type were rescued to media without hygromycin and transferred to the light. docl and doc0-6 were isolated in this screen. An additional 48,000 EMS-mutagenized $M_{2}$ seeds from pools that did not give rise to any surviving seedlings in the first screen were screened again in the same growth condition but using only $20 \mu \mathrm{g} / \mathrm{ml}$ of hygromycin B. doc2-1 and doc3 were isolated in this second screen. A third screen was done with 50,000 $\gamma$-ray mutagenized $\mathrm{M}_{2}$ seeds on $40 \mu \mathrm{g} / \mathrm{ml}$ of hygromycin $\mathrm{B}$ for 7 days in the dark. 
Li et al.

doc2-2, doc0-11, doc0-13, doc0-19 were isolated from this third screen. All mutant alleles were initially given the designation doc0-n. Individual loci were later numbered docil, doci2, doci3, etc., as they were mapped. All of the analyses with the three doc mutants presented in this study were performed with mutant lines that have been backcrossed to the wild type and then repeatedly selected for the mutant phenotype (hygromycin resistant and high GUS activity in the dark).

\section{Northern hybridizations, RNase protection, and GUS assays}

RNA was isolated using the acid guanidium thiocyanate-phenol extraction method as described (Chomczynski and Sacchi 1987). RNA was separated on formaldehyde-containing agarose gels as described in Ausubel et al. (1987), except $0.22 \mathrm{M}$ formaldehyde was used both in the gel and in the running buffer. RNA was then blotted onto Nytran nylon membranes /Schleicher \& Schuell) and immobilized to the membrane by UV cross-linking and baking. The membrane was prehybridized for $0.5 \mathrm{hr}$ at $60^{\circ} \mathrm{C}$ in a solution containing $1 \% \mathrm{BSA}, 1 \mathrm{mM}$ EDTA, $0.5 \mathrm{M} \mathrm{NaHPO}_{4}$ and $7 \%$ SDS. Hybridization with specific probes was done in the same solution at $60^{\circ} \mathrm{C}$ for $16 \mathrm{hr}$. The membrane was then washed three times at $65^{\circ} \mathrm{C}$ with $0.1 \times \mathrm{SSC}$ and $0.5 \%$ SDS. The membrane was exposed to $\mathrm{X}$-ray film with an intensifying screen. Quantification of samples was done by scanning the autoradiographs with an LKB UltroScan XL densitometer. The probe for $C A B$ mRNAs was generated from a plasmid containing the $C A B 2$ genomic DNA (Leutwiler et al. 1986) and the probes for $R B C S$ mRNA and rRNA were generated from plasmids as described elsewhere (Chory et al. 1989).

RNase protection experiments were done as described by Brusslan and Tobin (1992). Specific GUS activities of seedlings were analyzed with protein extracts from whole seedlings using the fluorometric assay as described by Jefferson (1987).

\section{Mapping of the three doc loci}

Mapping was done using the codominant ecotype-specific PCRbased markers as described (Konieczny and Ausubel 1993). docl was outcrossed to the ecotype Niederzenz. DNA from 154 individual $F_{2}$ plants ( 308 chromatids) that have the phenotype of docl (reduced apical dominance) were tested. From these samples, docl was found to be $-36 \mathrm{cM}$ from the marker GapA on chromosome III. docl was then crossed to the hy2 mutant in the Landsberg erecta ecotype. From $48 \mathrm{~F}_{2}$ DNA samples $(96$ chromatids), docl was mapped to $\sim 3 \mathrm{~cm}$ from the marker GapC and $2 \mathrm{cM}$ from an RFLP marker in the myb4 gene on chromosome III. $d o c 2$ and $d o c 3$ were both outcrossed to a multiply marked Landsberg line (an-er-py-g11-cer4). Mutants in the $\mathrm{F}_{2}$ progeny were identified with the phenotype of hygromycin resistance. Linkage of doc2 with the DFR ( $t$ t 4 ) marker on chromosome $\mathrm{V}$ was based on the analysis of $252 F_{2}$ chromatids and linkage of doc3 with $A D H$ and $G a p B$ on chromosome I was based on the analysis of $166 \mathrm{~F}_{2}$ chromatids. Location of doc0-6 was calculated based on its linkage to the pOCA107 transgene. doc0-6 was crossed to the Columbia ecotype. The $\mathrm{F}_{2}$ progeny showed a ratio of 2:1 of mutant/wild type, indicating a linkage with the transgene /a ratio of $9: 7$ is expected if the mutation is independent of the transgene, and a ratio of $3: 1$ is expected if the mutation is in the transgene).

\section{Construction of double mutants}

Double mutants were constructed by manual cross-pollination, by use of plants homozygous for the desired individual single mutation. The resulting $F_{1}$ plants were allowed to self pollinate.
In the $F_{2}$ generation, because no new phenotype was observed, mutants with phenotypes of each parent (det or doc) were isolated and their seeds harvested individually. The seeds from these plants were planted and scored for phenotypic segregation in the $F_{3}$ generation. Mutants that are heterozygous for the other mutation should segregate out 1 of 4 double mutants in the $F_{3}$ seedlings, and these double mutants could be identified if the phenotype of the double mutant is different from the original $F_{2}$ mutant plant. For all of the doc mutants isolated from the $F_{2}$ generation, more than half of them segregated out 1 of 4 seedlings with the det phenotype in the $\mathrm{F}_{3}$ generation. These det seedlings from the $\mathrm{F}_{2}$ doc plants were thus considered double mutants for the respective det and doc mutations. All the det plants isolated from the $F_{2}$ generation never segregated any plants with a new phenotype in the $\mathrm{F}_{3}$ generation.

\section{Acknowledgments}

We thank Kevin Culligan for assistance in mapping doc2 and doc3. We are grateful to Drs. Elaine Tobin, Judy Brusslan, and Judy Callis for the protocol and plasmids for making the $C A B 1$ and $U B Q 3$ probes for the RNase protection experiment. We also thank Kim Hanson, Drs. Peter Doenner, Jacky Pallas, Alan Pepper, Jason Reed, and Tracy Washburn for critical reading of this manuscript and all the members of the Chory laboratory for helpful discussions during the course of this work. This research was supported by grants from the Department of Energy and the National Science Foundation (to J.C.). Hsou-min $\mathrm{Li}$ is a fellow of the Samuel Roberts Noble Foundation. Lothar Altschmied was a fellow of Deutsche Forschungsgemeinschaft and The International Human Frontier Science Program Organization.

The publication costs of this article were defrayed in part by payment of page charges. This article must therefore be hereby marked "advertisement" in accordance with 18 USC section 1734 solely to indicate this fact.

\section{References}

Ausubel, F.M., R. Brent, R.E. Kingston, D.D. Moore, J.G. Seidman, J.A. Smith, and K. Struhl. 1987. Current protocols in molecular biology. Greene Publishing Associates, New York.

Brusslan, J.A. and E.M. Tobin. 1992. Light-independent developmental regulation of $c a b$ gene expression in Arabidopsis thaliana seedlings. Proc. Natl. Acad. Sci. 89: 7791-7795.

Brusslan, J.A., G.A. Karlin-Neumann, L. Huang, and E.M. Tobin. 1993. An Arabidopsis mutant with a reduced level of cab140 RNA is a result of cosuppression. Plant Cell 5: 667677.

Cabrera y Poch, H.L., C.A. Peto, and J. Chory. 1993. A mutation in the Arabidopsis DET3 gene uncouples photoregulated leaf development from gene expression and chloroplast biogenesis. Plant $J$. 4: 671-682.

Castresana, C., I. Garcia-Luque, E. Alonso, V.S. Malik, and A.R. Cashmore. 1988. Both positive and negative regulatory elements mediate expression of a photoregulated $C A B$ gene from Nicotiana plumbaginifolia. EMBO I. 7: 1929-1936.

Chomczynski, P. and N. Sacchi. 1987. Single step method of RNA isolation by acid guanidium thiocyanate-phenol-chloroform extraction. Anal. Biochem. 162: 156-159.

Chory, J. 1991. Light signals in leaf and chloroplast development: Photoreceptors and downstream responses in search of a transduction pathway. New Biol. 3: 538-548.

1992. A genetic model for light-regulated seedling de- 
velopment in Arabidopsis. Development 115: 337-354

Chory, J. and C.A. Peto. 1990. Mutations in the DETl gene affect cell-type-specific expression of light-regulated genes and chloroplast development in Arabidopsis. Proc. Natl. Acad. Sci. 87: 8776-8780.

Chory, J., C.A. Peto, R. Feinbaum, L. Pratt, and F. Ausubel. 1989. Arabidopsis thaliana mutant that develops as a lightgrown plant in the absence of light. Cell 58: 991-999.

Chory, J., P. Nagpal, and C.A. Peto. 1991. Phenotypic and genetic analysis of $\operatorname{det} 2$, a new mutant that affects light-regulated seedling development in Arabidopsis. Plant Cell 3: 445-459.

Chory, J., L. Altschmied, H. Cabrera, H.-M. Li, and R. Susek. 1993. Genetic dissection of signal transdution pathways that regulate $\mathrm{CAB}$ gene expression. In Cellular communication in plants (ed. R.M. Amasino), pp. 57-62. Plenum Press, New York.

Chory, J., D. Reinecke, S. Sim, T. Washburn, and M. Brenner. 1994. A role for cytokinins in de-etiolation in Arabidopsis: det mutants have an altered response to cytokinins. Plant Physiol. (in press).

Deng, X.-W., T. Caspar, and P.H. Quail. 1991. cop1: A regulatory locus involved in light-controlled development and gene expression in Arabidopsis. Genes \& Dev. 5: 11721182.

Donald, R.G.K. and A.R. Cashmore. 1990. Mutation of either G box or I box sequences profoundly affects expression from the Arabidopsis rbcS-1A promoter. EMBO I. 9: 1717-1726.

Frances, S., M.J. White, M.D. Edgerton, A.M. Jones, R.C. Elliott, and W.F. Thompson. 1992. Initial characterization of a pea mutant with light-independent photomorphogenesis. Plant Cell 4: 1519-1530.

Gidoni, D., P. Brosio, D. Bond-Nutter, J. Bedbrook, and P. Dunsmuir. 1989. Novel cis-acting elements in Petunia Cab gene promoters. Mol. Gen. Genet. 215: 337-344.

Gilmartin, P.M., L. Sarokin, J. Memelink, and H.-H. Chua. 1990. Molecular light switches for plant genes. Plant Cell 2: 369-378.

Gilmartin, P.M., J. Memelink, K. Hiratsuka, S. Kay, and N.-H. Chua. 1992. Characterization of a gene encoding a DNA binding protein with specificity for a light-responsive element. Plant Cell 4: 839-849.

Hou, Y., A.G.v.Arnim, and X.-W. Deng. 1993. A new class of Arabidopsis constitutive photomorphogenic genes involved in regulating cotyledon development. Plant Cell 5: 329-339.

Jefferson, R.A. 1987. Assaying chimeric genes in plants: The GUS gene fusion system. Plant Mol. Biol. Reporter 5: 387405.

Karlin-Neumann, G.A., L. Sun, and E.M. Tobin. 1988. Expression of light-harvesting chlorophyll $a / b$-protein genes is phytochrome-regulated in etiolated Arabidopsis thaliana seedlings. Plant Physiol. 88: 1323-1331.

Kaufman, L.S., W.F. Thompson, and W.R. Briggs. 1984. Different red light requirements for phytochrome-induced accumulation of $c a b$ and $r b c S$ RNA. Science 226: 1447-1449.

Konieczny, A. and F.M. Ausubel. 1993. A procedure for mapping Arabidopsis mutations using codominant ecotype-specific PCR-based markers. Plant I. 4: 403-410.

Krapp, A., B. Hofmann, C. Schafer, and M. an Stitt. 1993. Regulation of the expression of rbcS and other photosynthetic genes by carbohydrates: A mechanism for the "sink regulation" of photosynthesis. Plant J. 3: 817-828.

Leutwiler, L.S., E.M. Meyerowitz, and E.M. Tobin. 1986. Structure and expression of three light-harvesting chlorophyll $a / b$ binding protein genes in Arabidopsis thaliana. Nucleic Acids Res. 14: 4051-4064.
Li, H.-m., T. Washburn, and J. Chory. 1993. Regulation of gene expression by light. Curr. Opin. Cell Biol. 5: 455-460.

McGrath, J.M., W.B. Terzaghi, P. Sridhar, A.R. Cashmore, and E. Pichersky. 1992. Sequences of the fourth and fifth photosystem II type I chlorophyll $a / b$-binding protein genes of Arabidopsis thaliana and evidence for the presence of a full complement of the extended CAB gene family. Plant Mol. Biol. 19: 725-733.

Norris, S.R., S.E. Meyer, and J. Callis. 1993. The intron of Arabidopsis thaliana polyubiquitin genes is conserved in location and is a quantitative determinant of chimeric gene expression. Plant Mol. Biol. 21: 895-906.

Okubara, P.A., S.A. Williams, R.A. Doxsee, and E.M. Tobin. 1993. Analysis of genes negatively regulated by phytochrome action in Lemna gibba and identification of a promoter region required for phytochrome responsiveness. Plant Physiol. 101: 915-924.

Perisic, O. and E. Lam. 1992. A tobacco DNA binding protein that interacts with a light-responsive box II element. Plant Cell 4: 831-838.

Schindler, U., A.E. Menkens, H. Beckmann, J.R. Ecker, and A.R. Cashmore. 1992. Heterodimerization between light-regulated and ubiquitously expressed Arabidopsis GBF bZIP proteins. EMBO I. 11: 1261-1273.

Schulze-Lefert, P., M. Becker-André, W. Schulz, K. Hahlbrock, and J. Dangl. 1989. Functional architecture of the light-responsive chalcone synthase promoter from parsley. Plant Cell 1: 707-714.

Sheen J. 1990. Metabolic repression of transcription in higher plants. Plant Cell 2: 1027-1038.

Somerville, C.R. and W.L. Ogren. 1982. Isolation of photorespiration mutants in Arabidopsis thaliana. In Methods in chloroplast molecular biology (ed. M. Edelman, R.B. Hallick, and N.H. Chua), pp. 129-139. Elsevier Biomedical Press, New York.

Sun, L. and E.M. Tobin. 1990. Phytochrome-regulated expression of genes encoding light-harvesting chlorophyll $a / b$-protein in two long hypocotyl mutants and wild type plants of Arabidopsis thaliana. Photochem. Photobiol. 52: 51-56.

Susek, R. and J. Chory. 1992. A tale of two genomes: role of a chloroplast signal in coordinating nuclear and plastid genome expression. Aust. J. Plant Physiol. 19: 387-399.

Thompson, W.F. and M.J. White. 1991. Physiological and molecular studies of light-regulated nuclear genes in higher plants. Annu. Rev. Plant Physiol. Plant Mol. Bio. 42: 423466.

Wei, N. and X.-W. Deng. 1992. COP9: A new genetic locus involved in light-regulated development and gene expression in Arabidopsis. Plant Cell 4: 1507-1518.

Weisshaar, B., G.A. Armstrong, A. Bloch, O.D. Costa e Silva, and K. Hahlbrock. 1991. Light-inducible and constitutively expressed DNA-binding proteins recognizing a plant promoter element with functional relevance in light responsiveness. EMBO I. 10: 1777-1786. 


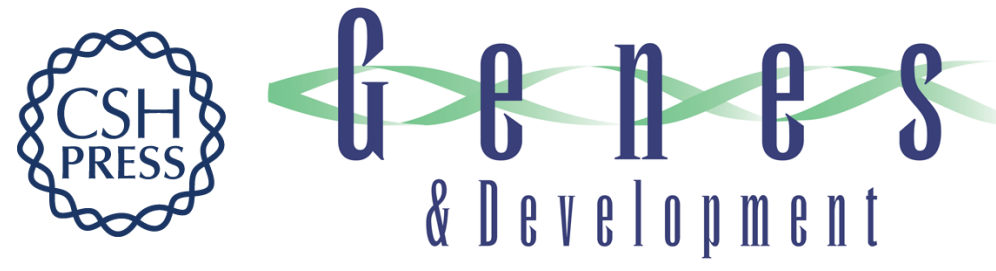

\section{Arabidopsis mutants define downstream branches in the phototransduction pathway.}

H M Li, L Altschmied and J Chory

Genes Dev. 1994, 8:

Access the most recent version at doi:10.1101/gad.8.3.339

References This article cites 36 articles, 17 of which can be accessed free at:

http://genesdev.cshlp.org/content/8/3/339.full.html\#ref-list-1

License

Email Alerting Receive free email alerts when new articles cite this article - sign up in the box at the top Service right corner of the article or click here.

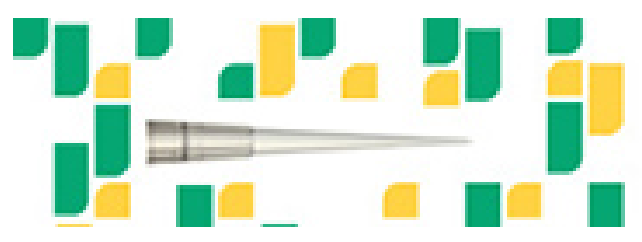

Focused on your science. 